\title{
Paxdaphnidines A and B, Novel Penta- and Tetracyclic Alkaloids from Daphniphyllum paxianum
}

Zha-Jun Zhan, Sheng-Ping Yang, and Jian-Min Yue*

State Key Laboratory of Drug Research, Institute of Materia Medica, Shanghai Institutes for Biological Sciences, Chinese Academy of Sciences, 555 Zu Chong Zhi Road, Zhangjiang Hi-Tech Park, Shanghai, 201203, P. R. China

jmyue@mail.shcnc.ac.cn.

\section{Supporting Information}

Figure S2. ${ }^{1} \mathrm{H}$ NMR spectrum of paxdaphnidineA (1) in $\mathrm{CD}_{3} \mathrm{OD}$.

Figure $\mathrm{S} 3 .{ }^{13} \mathrm{C}$ NMR spectrum of paxdaphnidineA (1) in $\mathrm{CD}_{3} \mathrm{OD}$.

Figure S4. ${ }^{13} \mathrm{C}$ NMR spectrum of paxdaphnidineA (1) in $\mathrm{CDCl}_{3}$.

Figure $\mathrm{S} 5 .{ }^{1} \mathrm{H}-{ }^{1} \mathrm{H}$ COSY spectrum of paxdaphnidineA (1) in $\mathrm{CD}_{3} \mathrm{OD}$.

Figure S6. HSQC spectrum of paxdaphnidine $\mathrm{A}$ (1) in $\mathrm{CD}_{3} \mathrm{OD}$ (20-50 ppm).

Figure $\mathrm{S} 7$. HSQC spectrum of paxdaphnidine $\mathrm{A}$ (1) in $\mathrm{CD}_{3} \mathrm{OD}$ (50-58 ppm).

Figure S8. HMBC spectrum of paxdaphnidineA (1) in $\mathrm{CD}_{3} \mathrm{OD}$.

Figure S9. NOESY spectrum of paxdaphnidineA (1) in $\mathrm{CD}_{3} \mathrm{OD}$.

Figure S10. EI MS spectrum of paxdaphnidineA (1).

Figure S11. ${ }^{1} \mathrm{H}$ NMR spectrum of paxdaphnidine $\mathrm{B}(2)$ in $\mathrm{CD}_{3} \mathrm{OD}$.

Figure $\mathrm{S} 12 .{ }^{13} \mathrm{C}$ NMR spectrum of paxdaphnidine $\mathrm{B}(2)$ in $\mathrm{CD}_{3} \mathrm{OD}$.

Figure $\mathrm{S} 13 .{ }^{1} \mathrm{H}^{-1} \mathrm{H}$ COSY spectrum of paxdaphnidine $\mathrm{B}(2)$ in $\mathrm{CD}_{3} \mathrm{OD}$.

Figure S14. HSQC spectrum of paxdaphnidine $\mathrm{B}(2)$ in $\mathrm{CD}_{3} \mathrm{OD}$.

Figure $\mathrm{S} 15$. $\mathrm{HMBC}$ spectrum of paxdaphnidine $\mathrm{B}(2)$ in $\mathrm{CD}_{3} \mathrm{OD}$.

Figure S16. NOESY spectrum of paxdaphnidine $\mathrm{B}(2)$ in $\mathrm{CD}_{3} \mathrm{OD}$.

Figure S17. EI MS spectrum of paxdaphnidine B (2).

General Procedures S18. 
S2

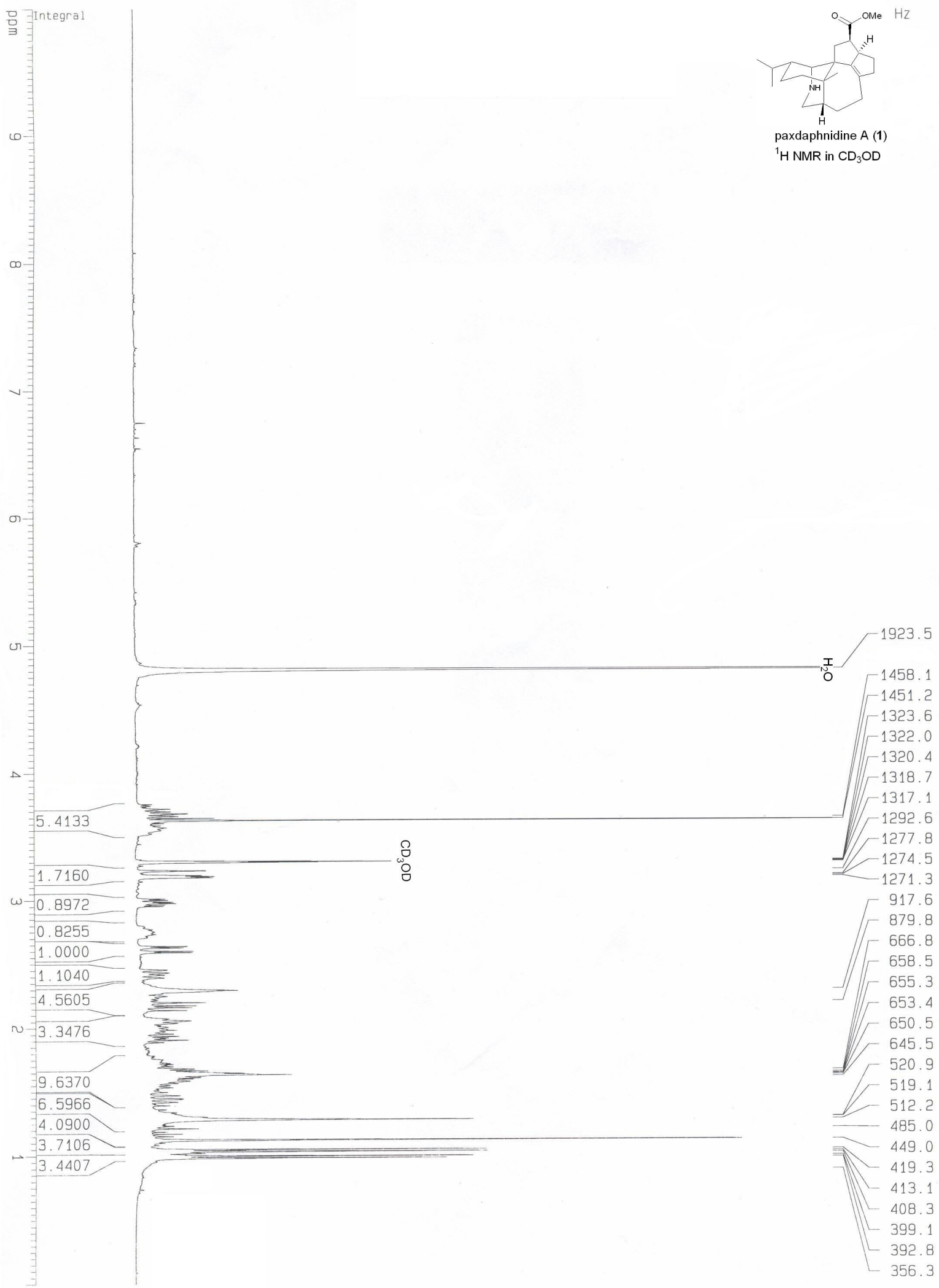


S3

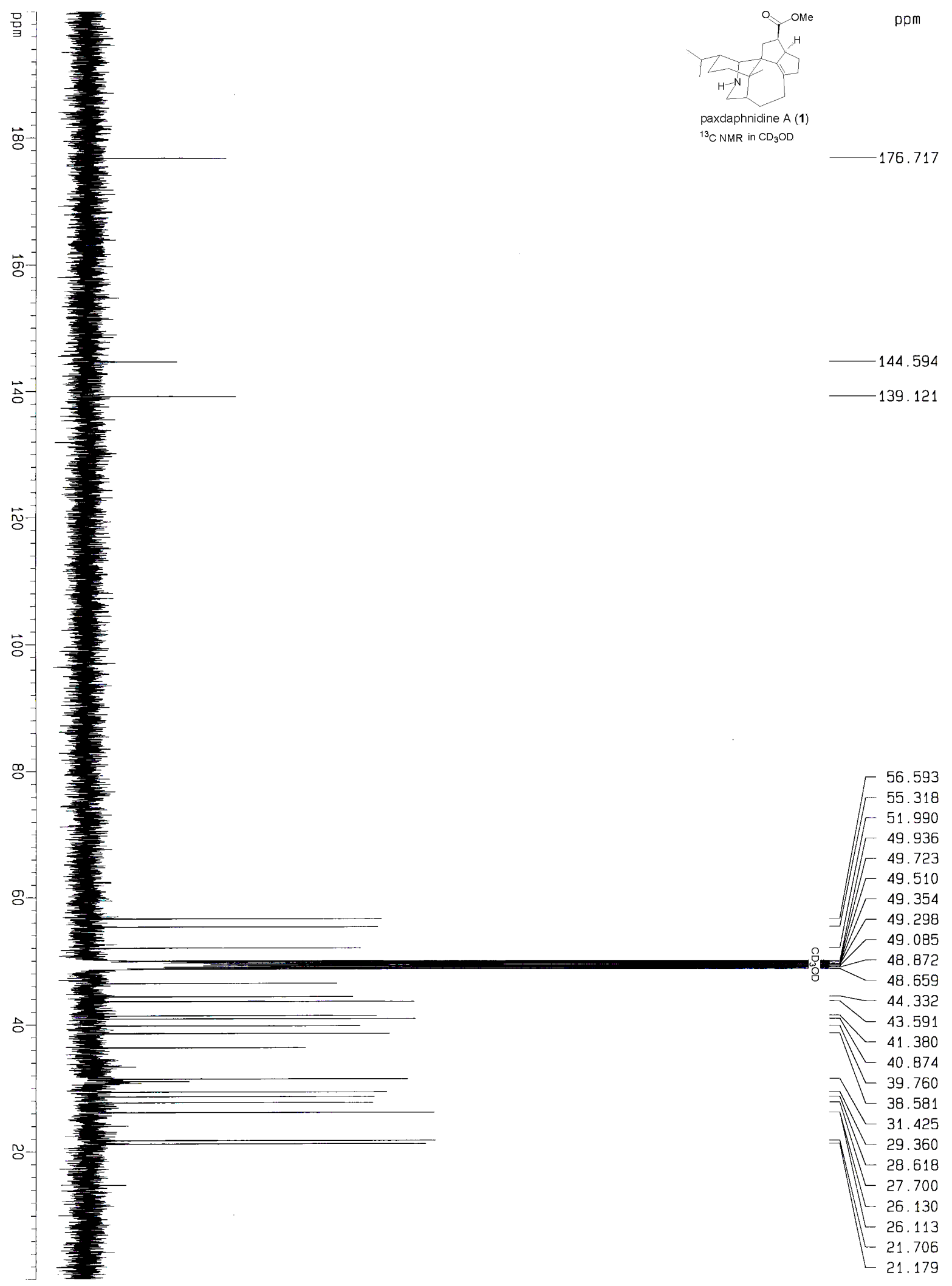



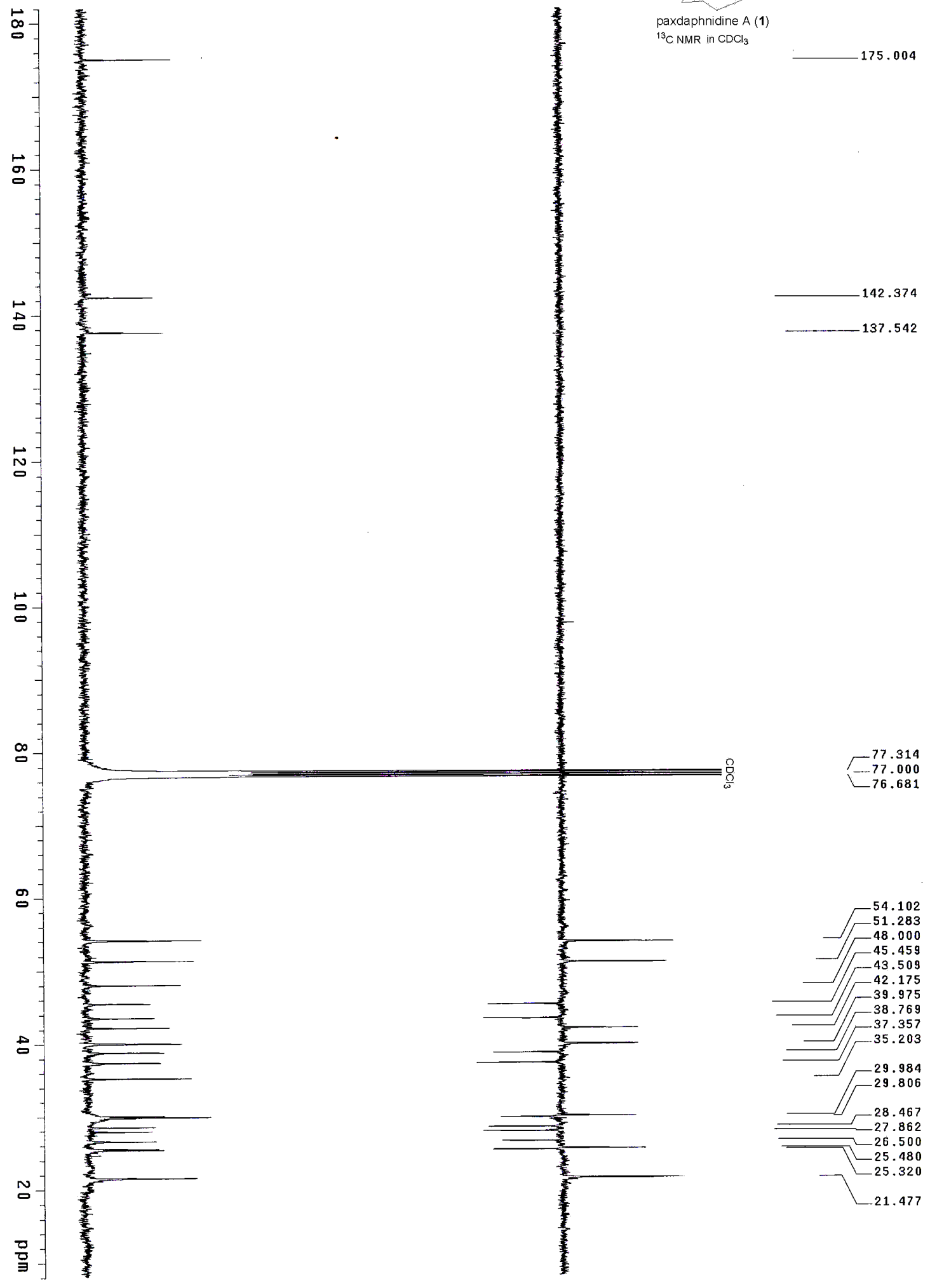

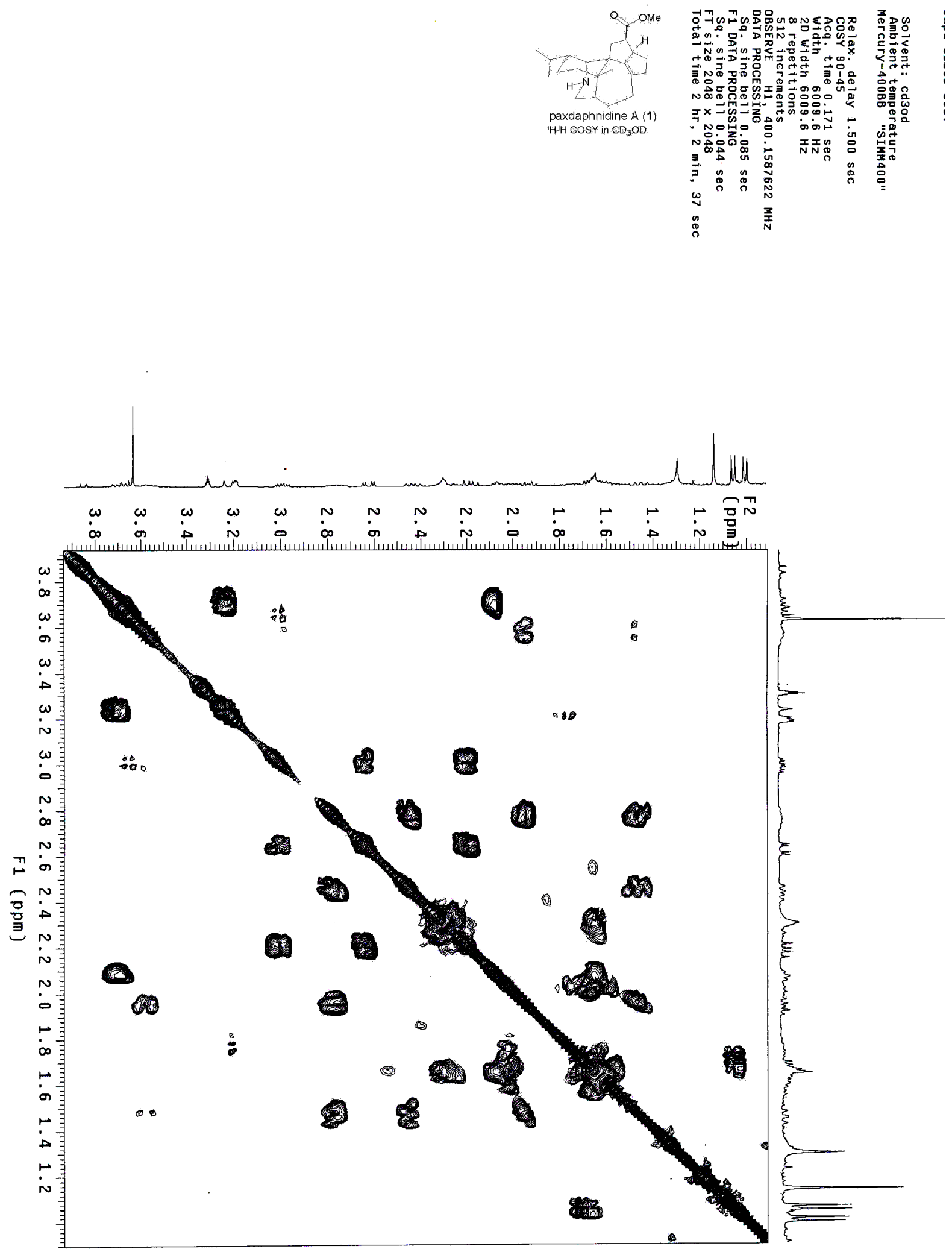


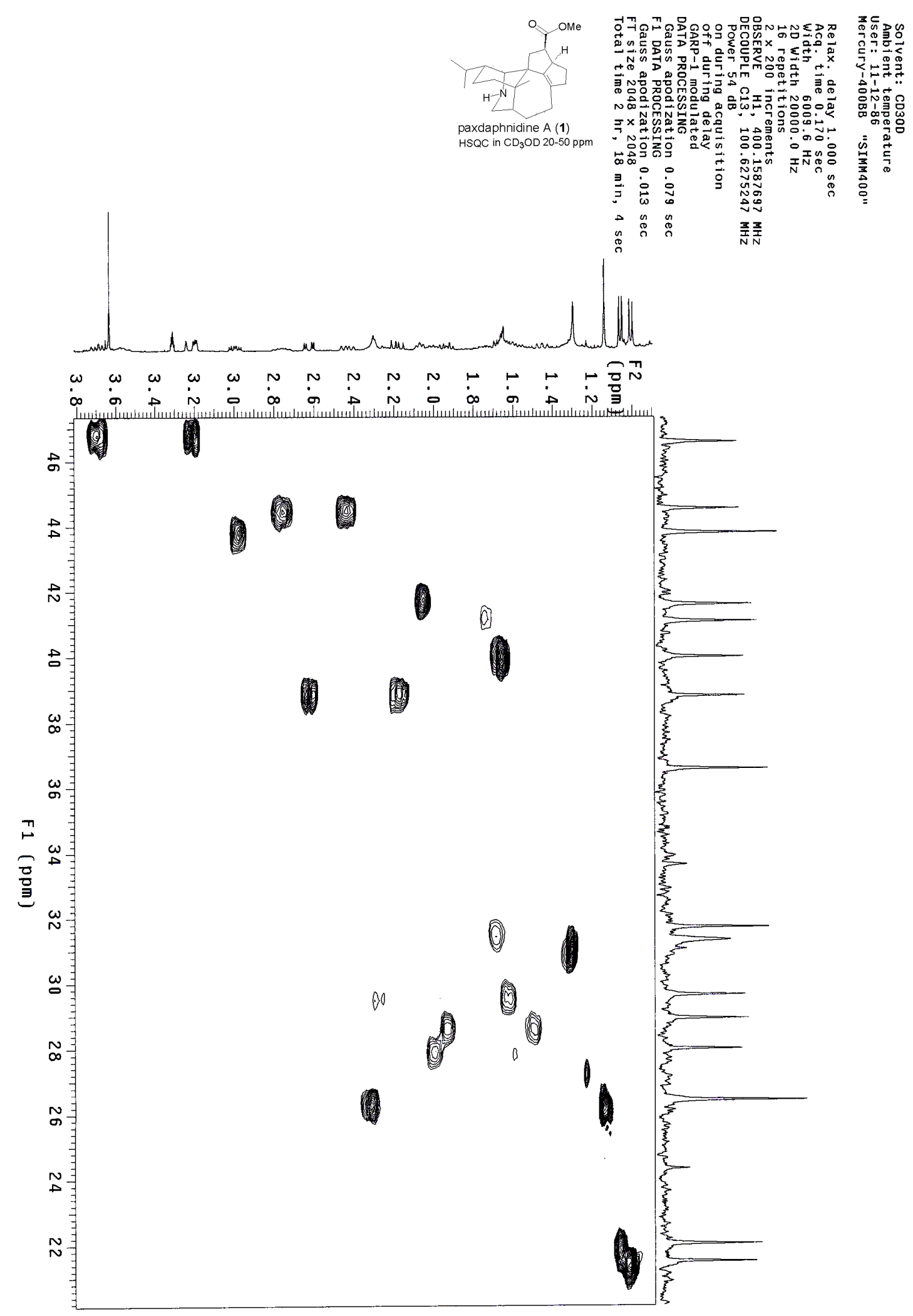




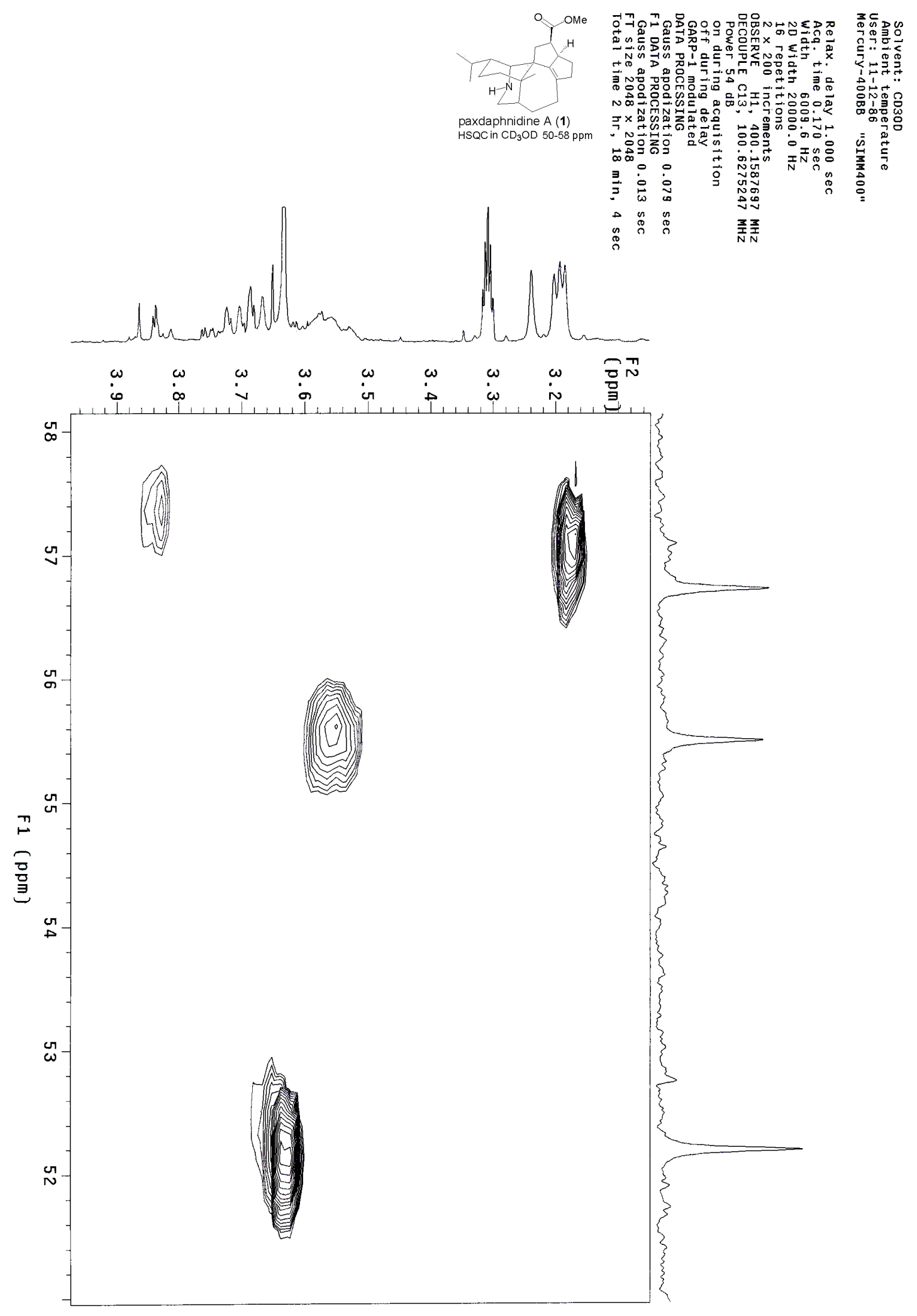


S8

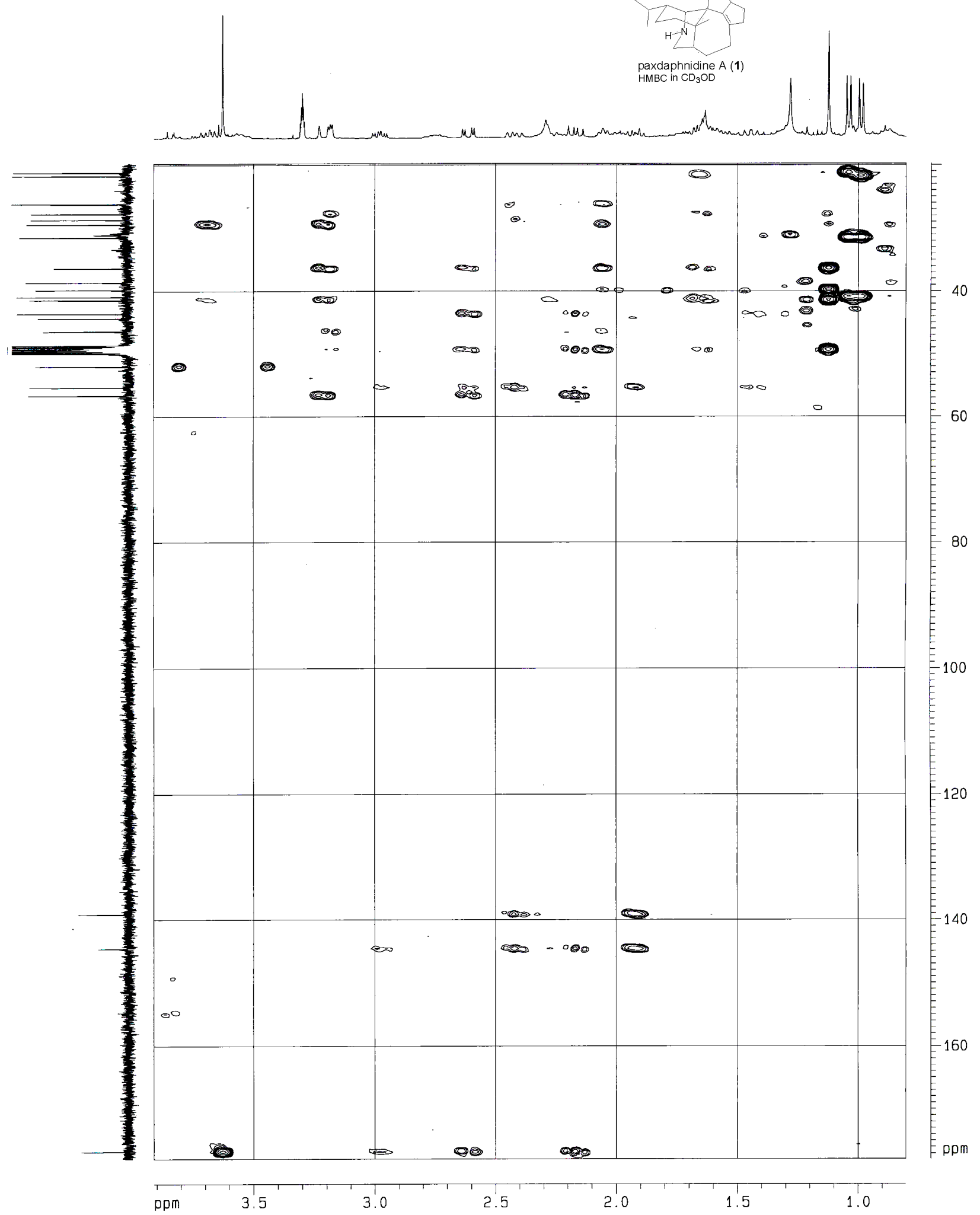




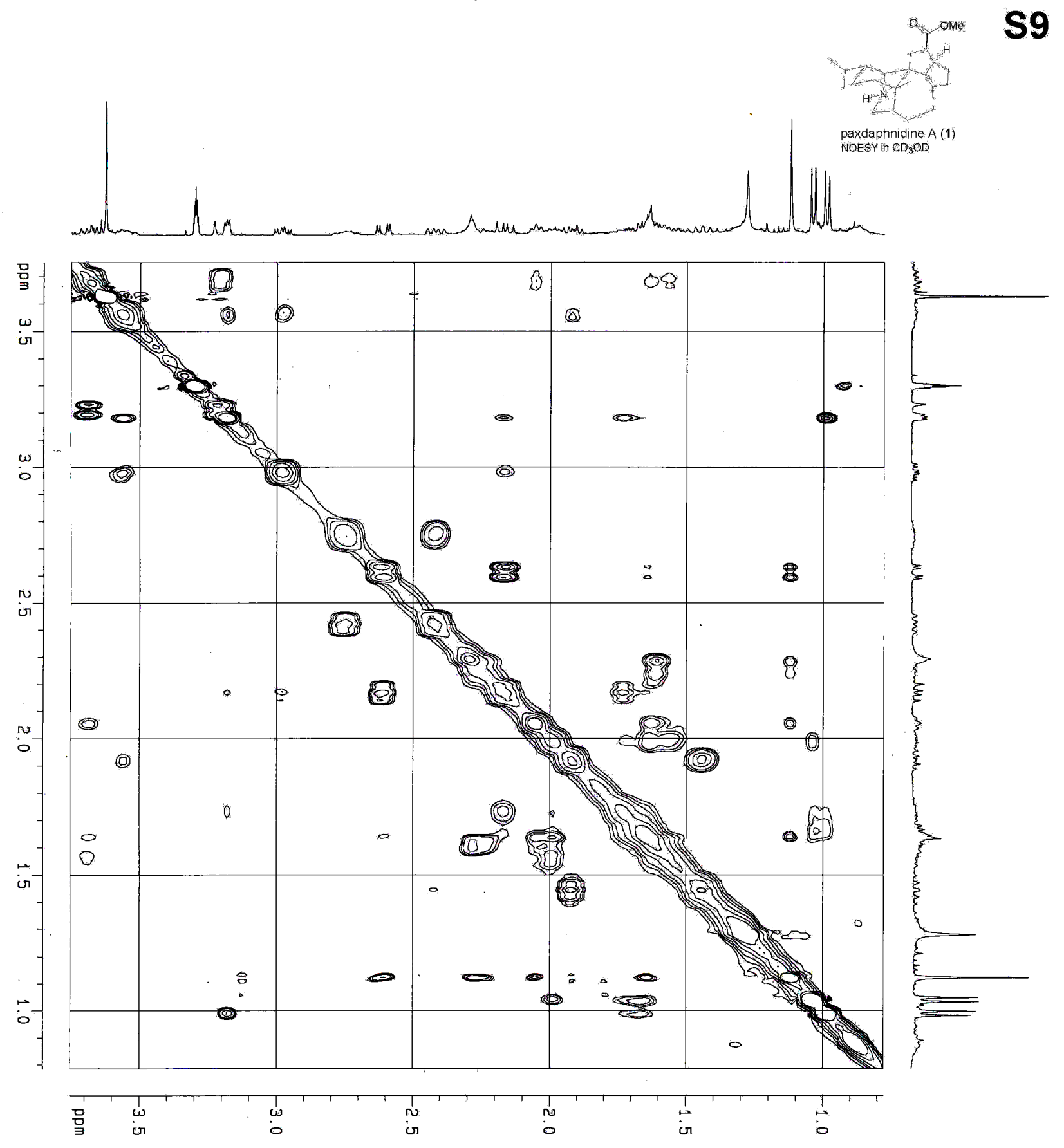




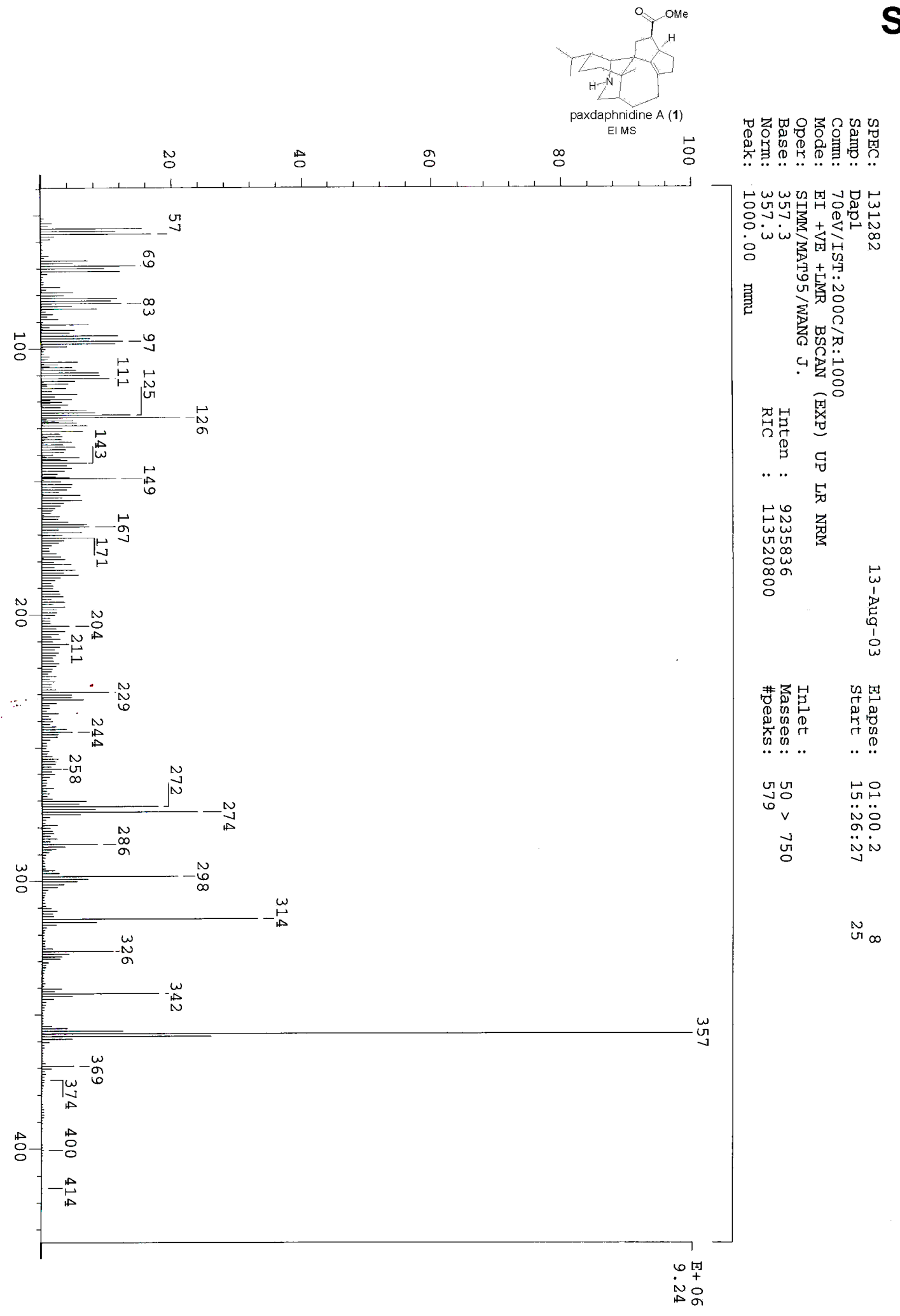


S11

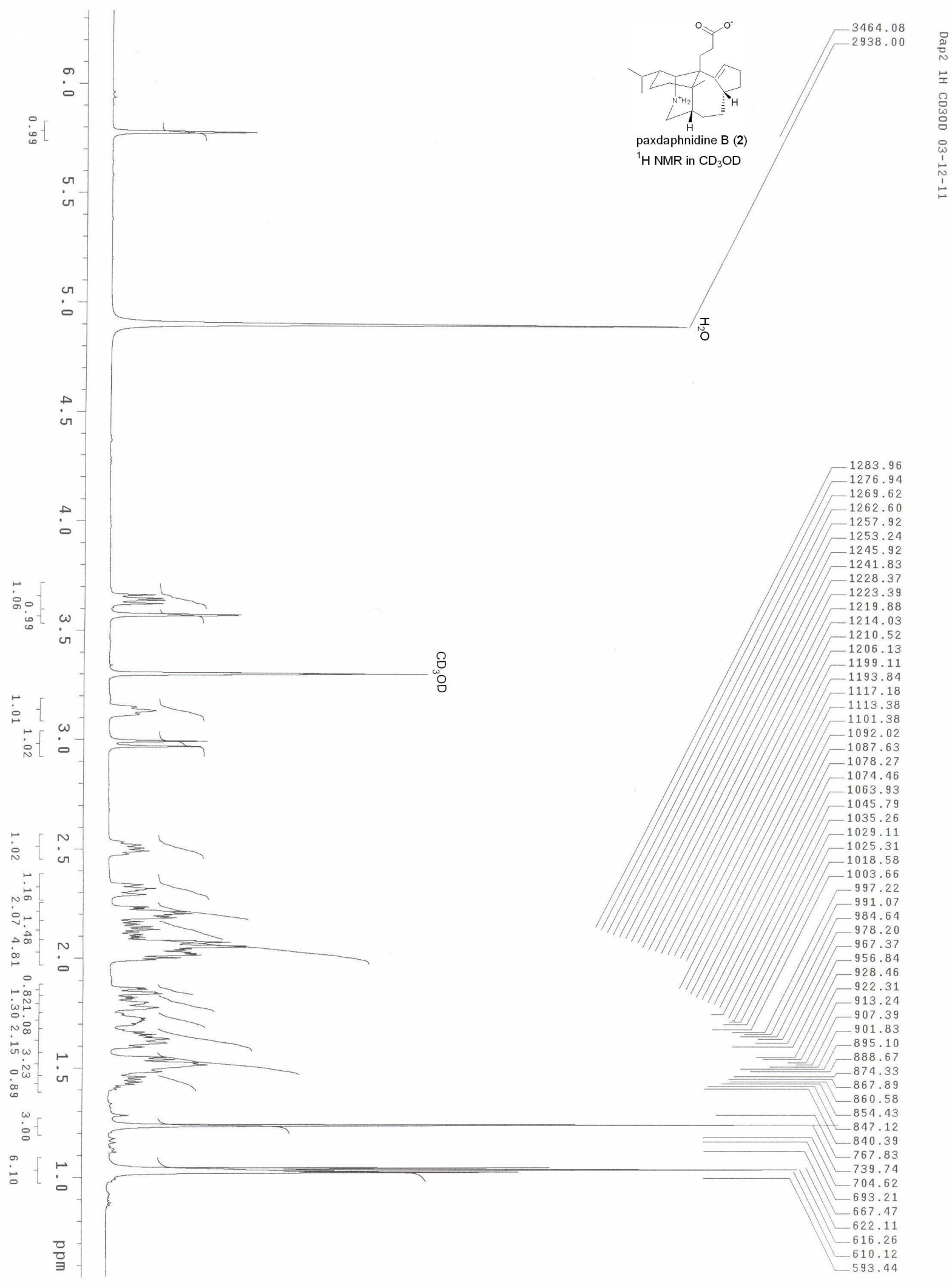


S12
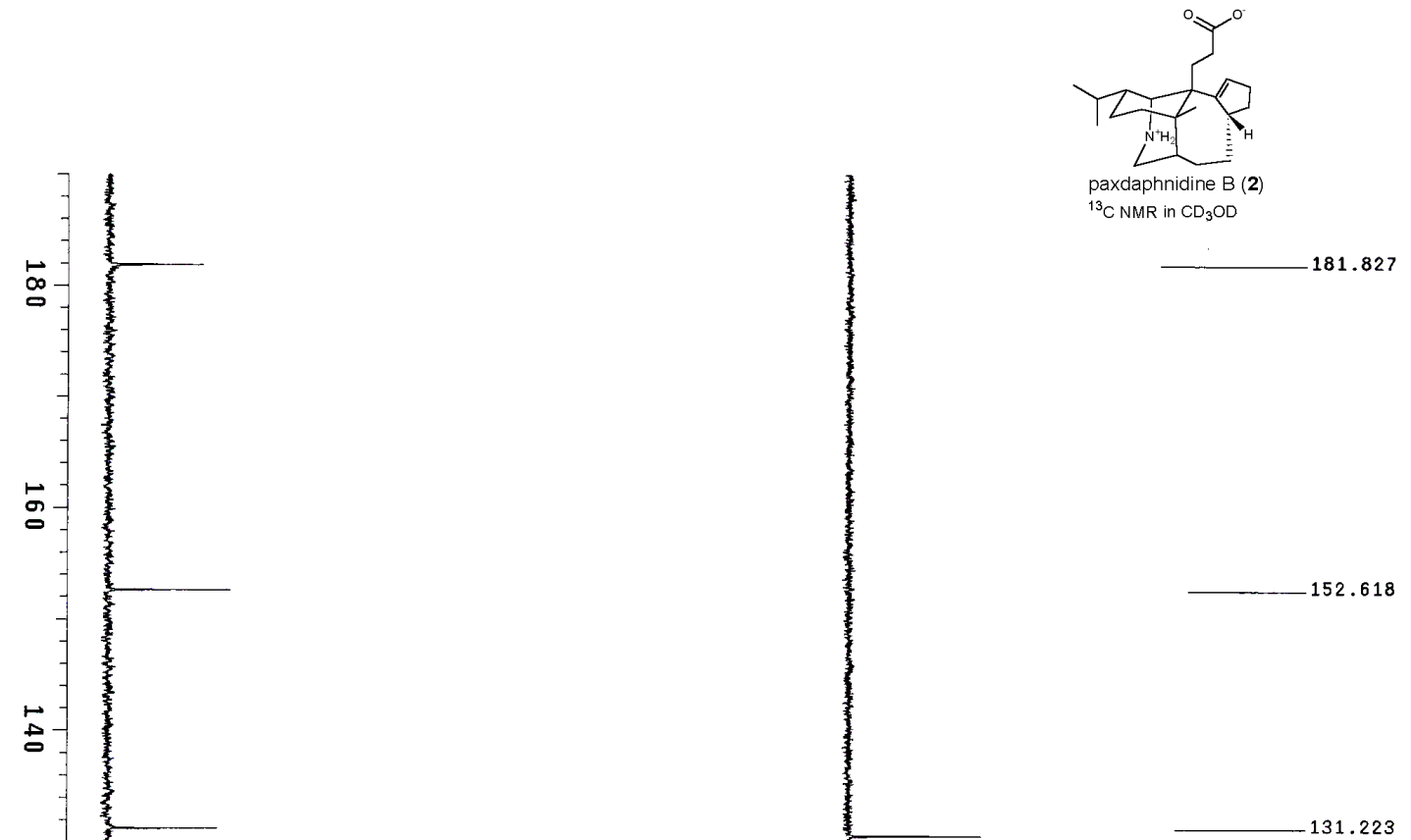

$\stackrel{\circ}{\circ}$

$\stackrel{\infty}{\circ}$

占-

․․․

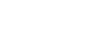

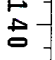

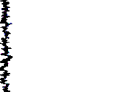

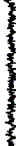

$\infty-$

-
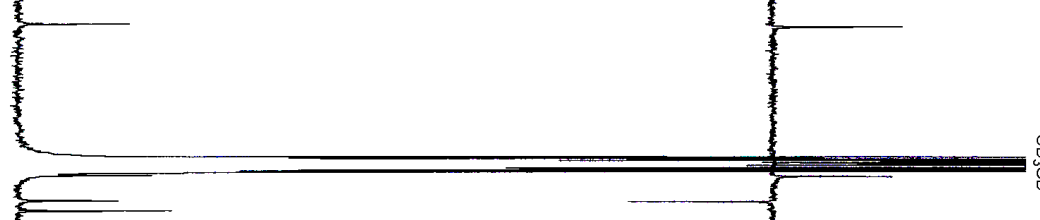

-

뭉
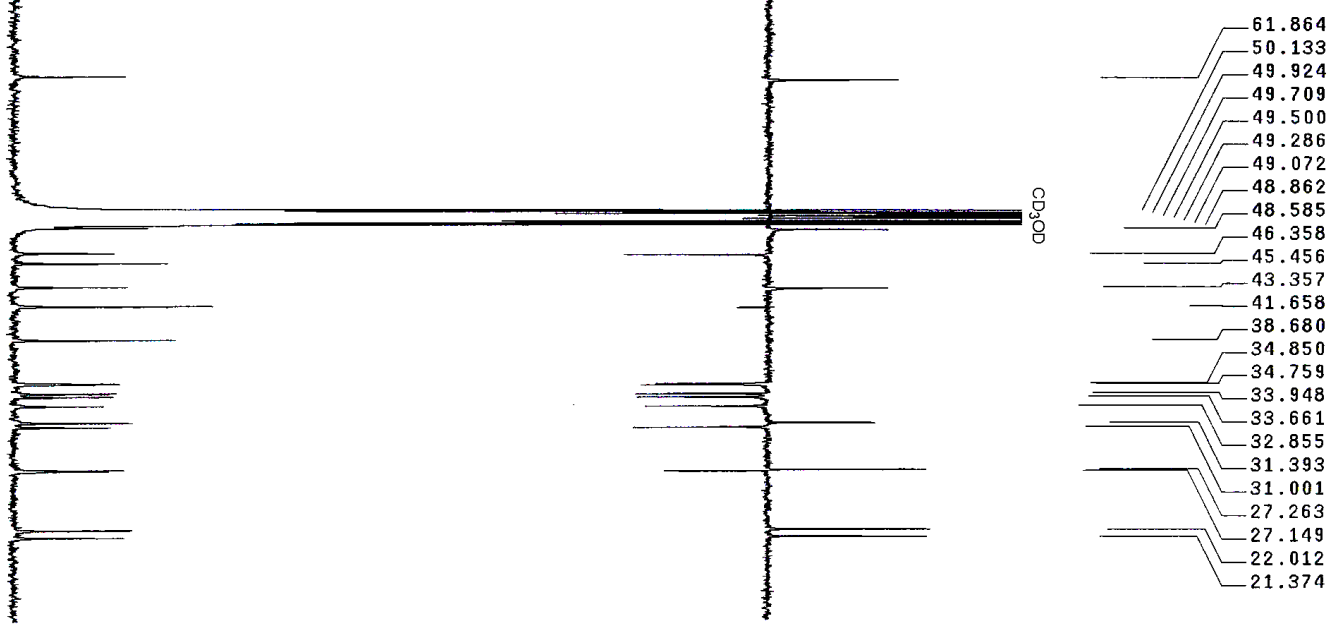
S13
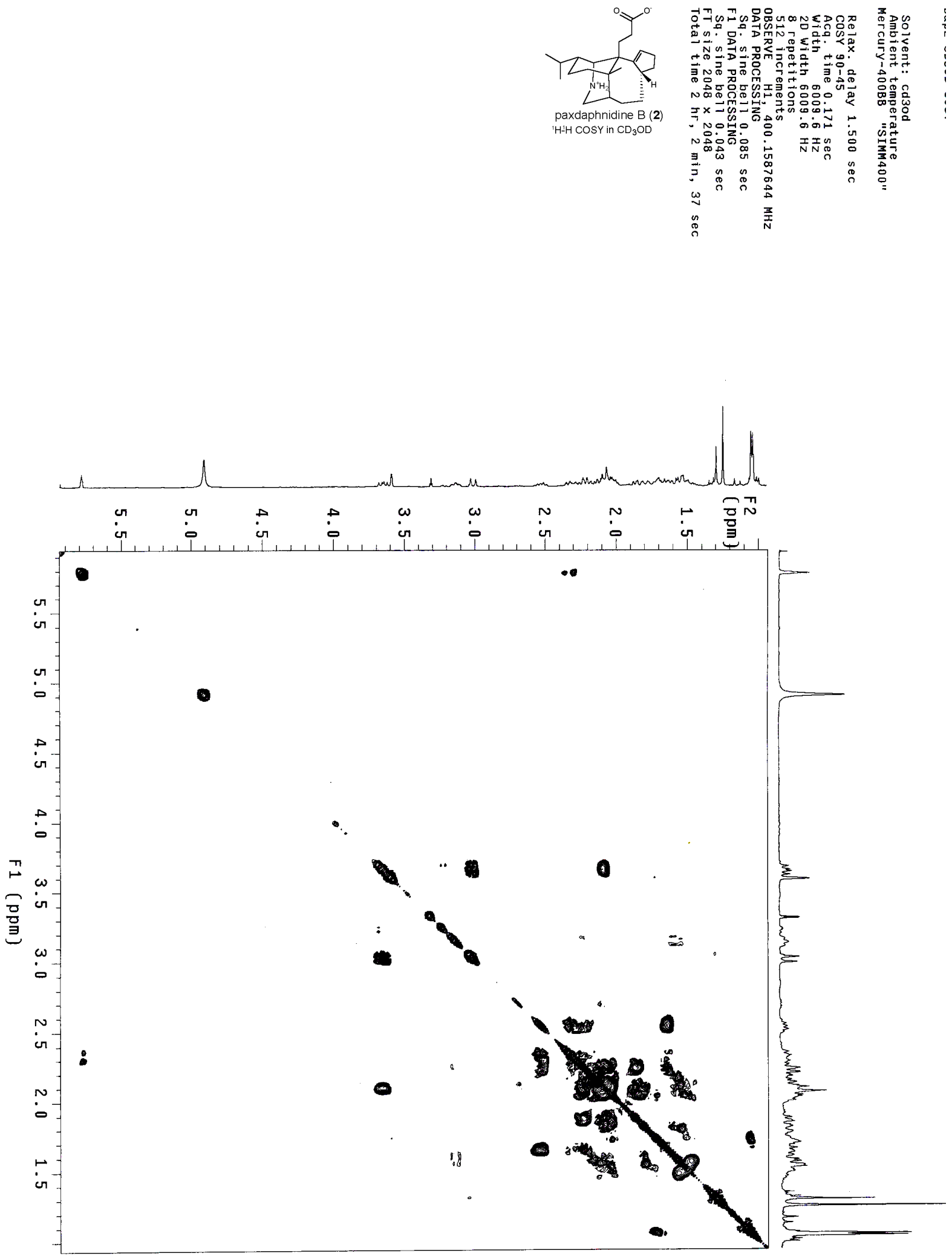


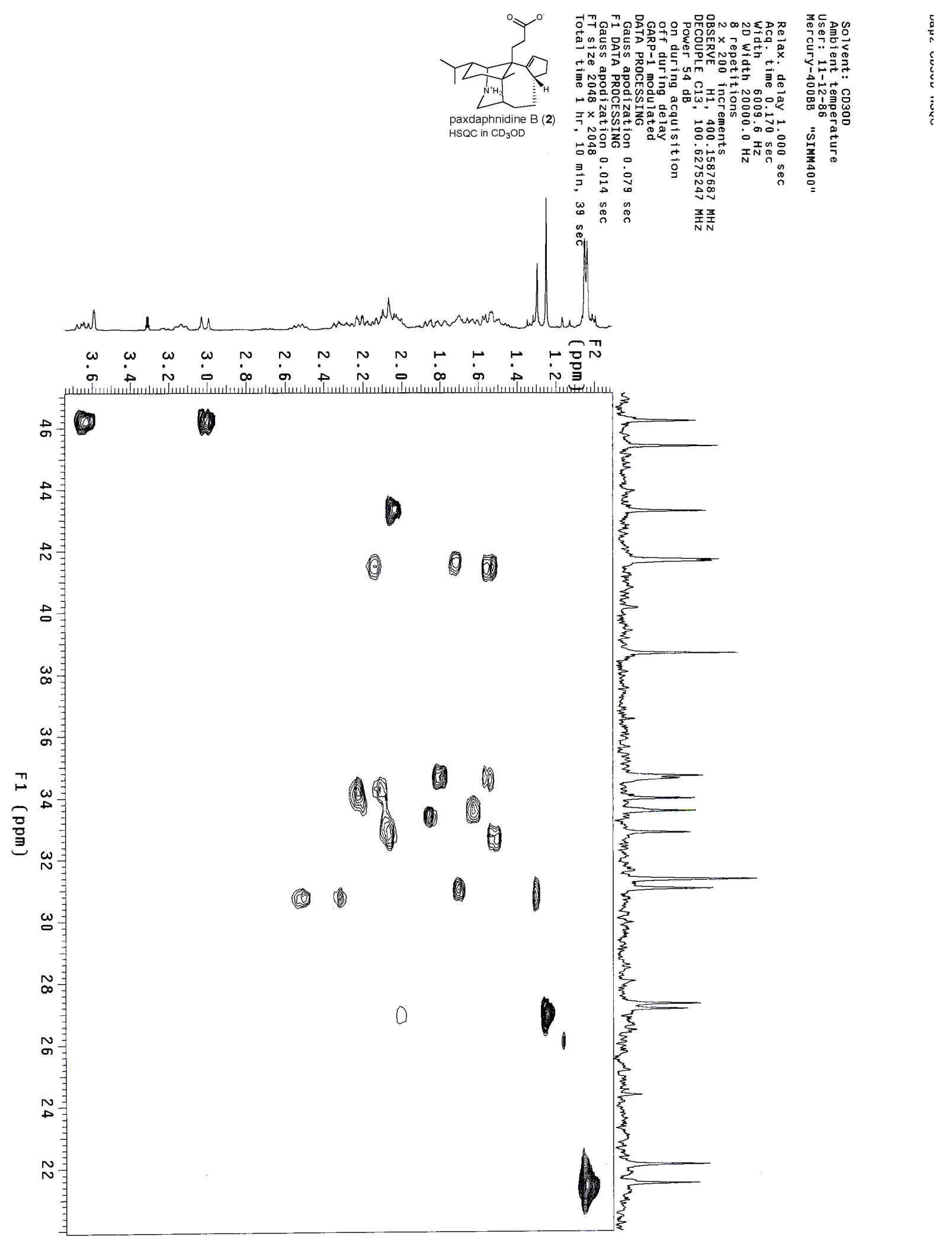




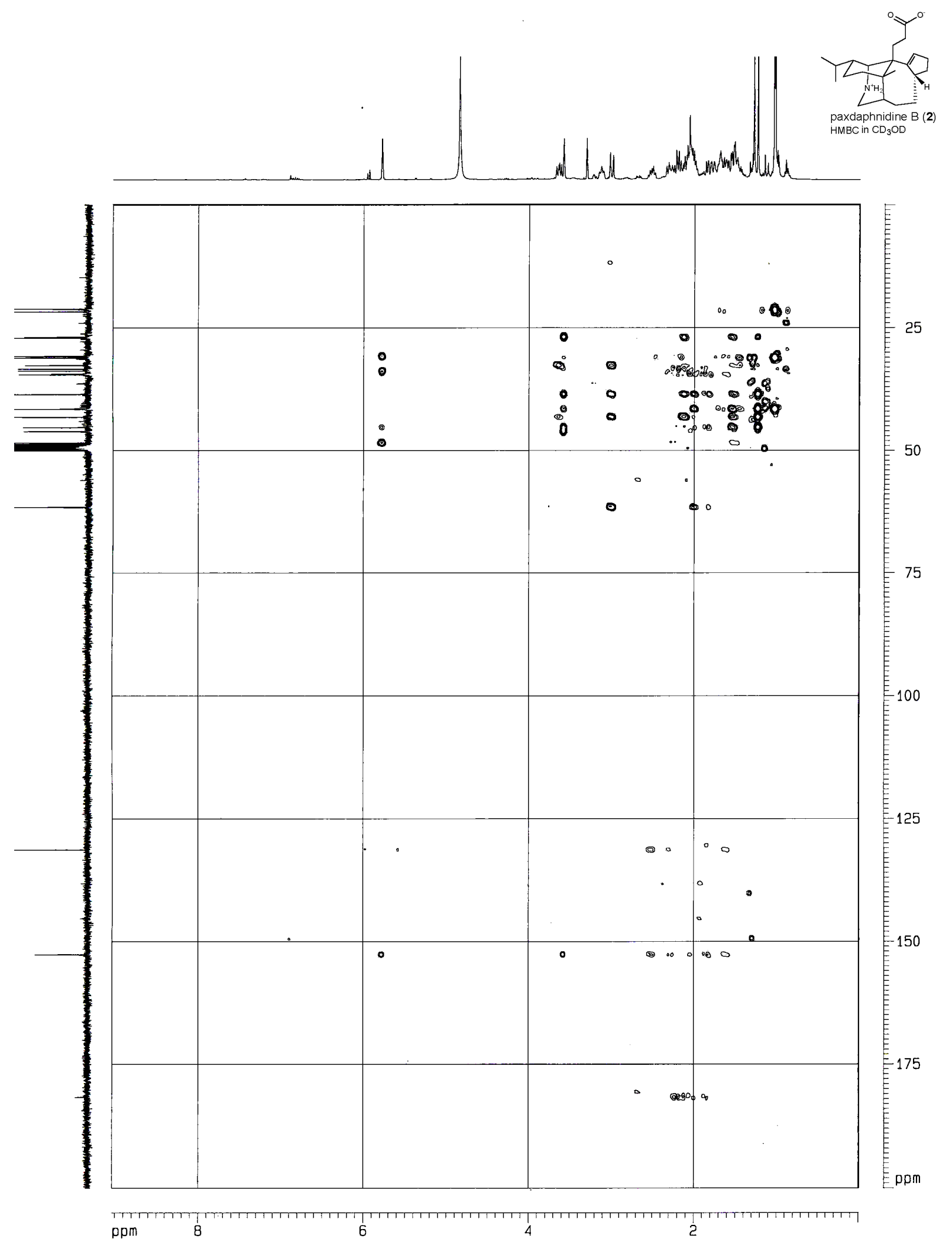




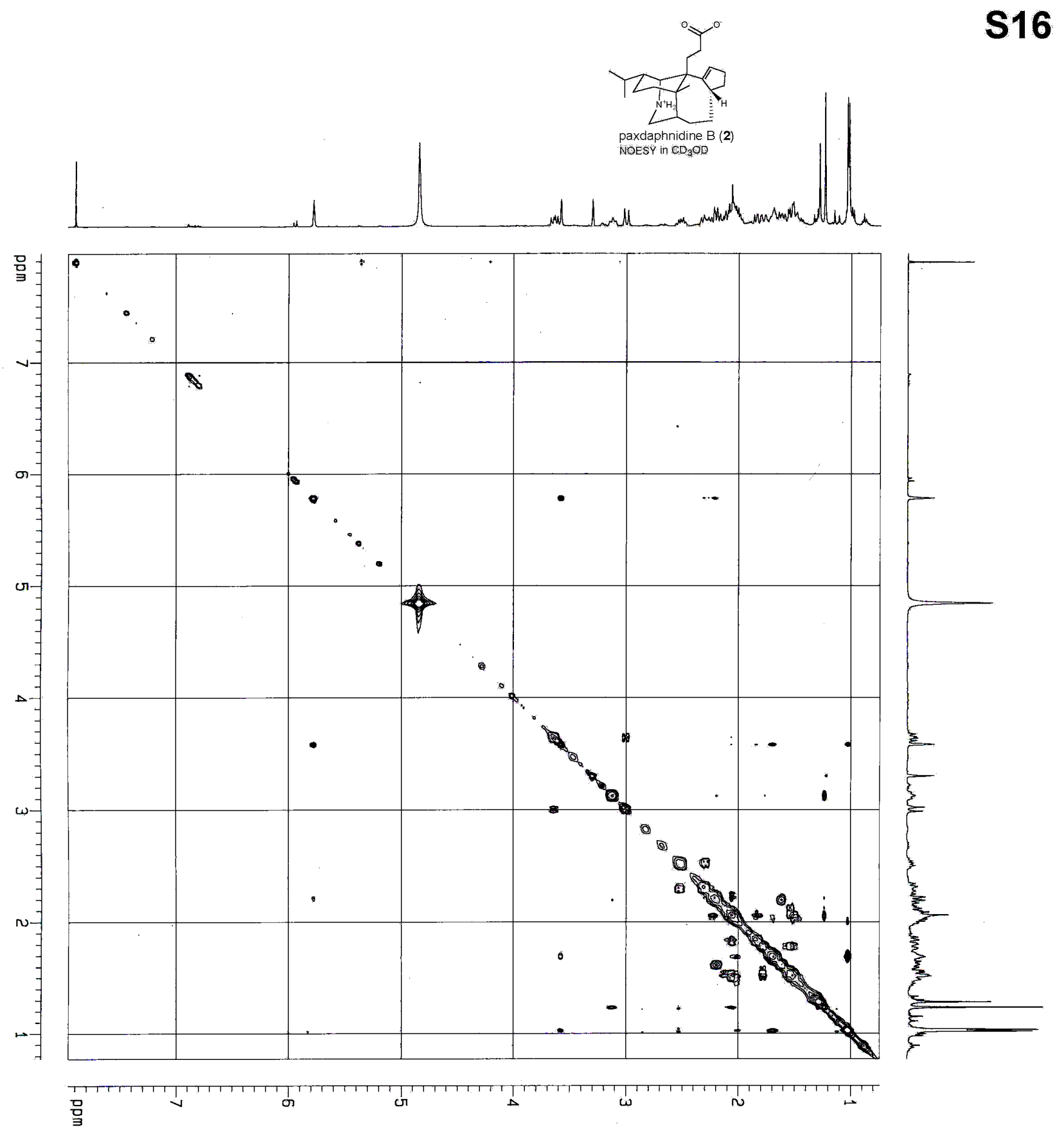




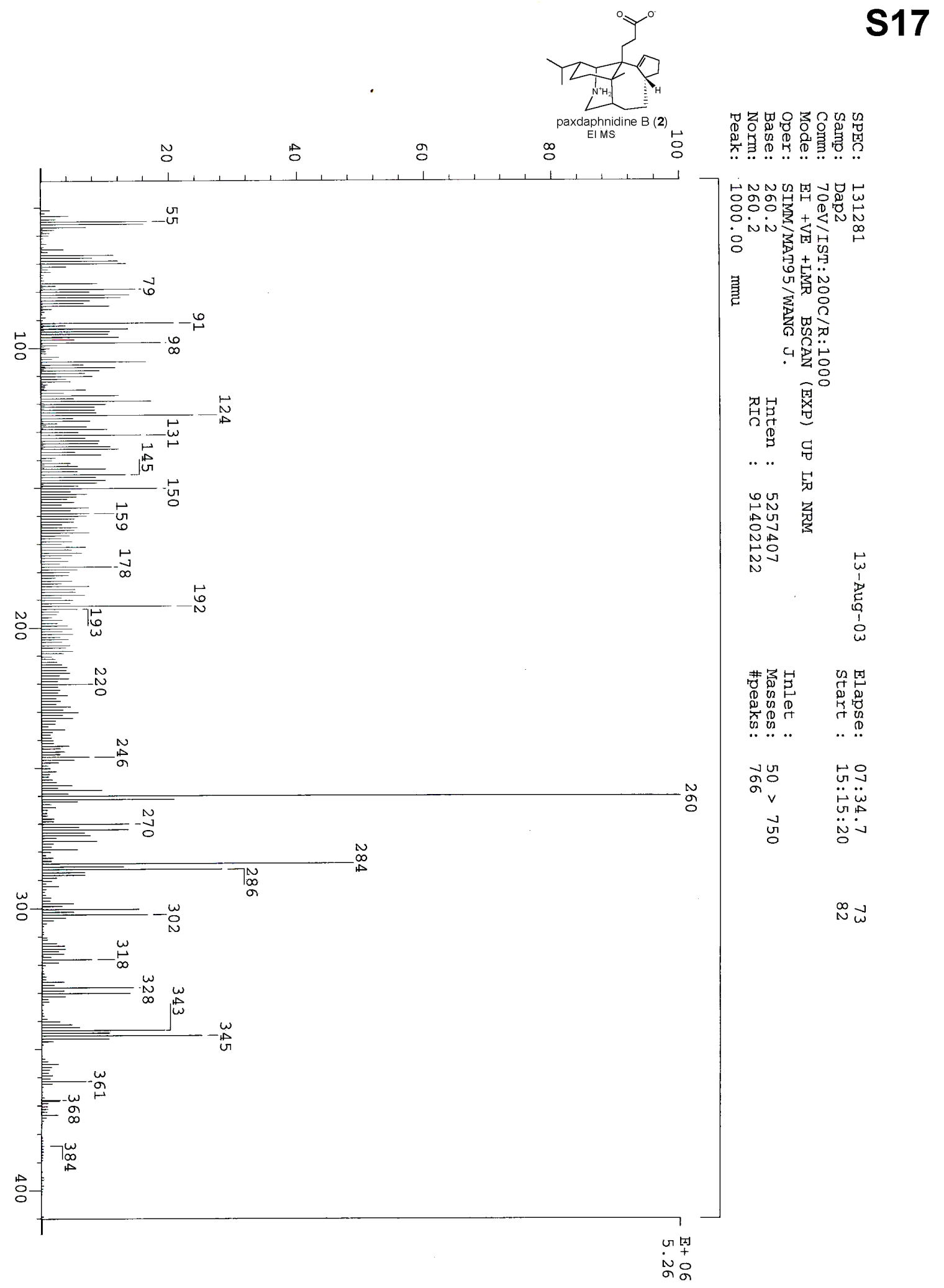


General Procedures. Melting point was recorded on Fisher-Johns melting point apparatus and uncorrected. Optical rotations were determined on a Perkin-Elmer 341 polarimeter. IR spectra were measured on a Perkin-Elmer 577 spectrometer. NMR spectra were made on a Bruker AM-400 and Bruker AM-600 spectrometer with TMS as internal standard. Standard pulse sequences were employed to obtain ${ }^{1} \mathrm{H}-{ }^{1} \mathrm{H}$ COSY, HMBC, HSQC and NOESY. EIMS $(70 \mathrm{eV})$ was carried out on a Finnigan MAT 95 mass spectrometer instrument. All solvents used were of analytical grade (Shanghai Chemical Plant). Silica gel (230-400 mesh) was used for column chromatography, and pre-coated silica gel $\mathrm{GF}_{254}$ plates (Qingdao Haiyang Chemical Plant) were used for TLC. Sephadex LH-20 was also used for column chromatograph. 